\title{
Joint Dynamic Radio Resource Allocation and Mobility Load Balancing in 3GPP LTE Multi-Cell Network
}

\author{
Feng LI, Lina GENG, Shihua ZHU \\ School of Electronics and Information Engineering, Xi'an Jiaotong University, Xianning Street, 710049 Xi'an, China \\ 1f1981@mail.xjtu.edu.cn, geng_lina@126.com,szhu@mail.xjtu.edu.cn
}

\begin{abstract}
Load imbalance, together with inefficient utilization of system resource, constitute major factors responsible for poor overall performance in Long Term Evolution (LTE) network. In this paper, a novel scheme of joint dynamic resource allocation and load balancing is proposed to achieve a balanced performance improvement in 3rd Generation Partnership Project (3GPP) LTE SelfOrganizing Networks (SON). The new method which aims at maximizing network resource efficiency subject to intercell interference and intra-cell resource constraints is implemented in two steps. In the first step, an efficient resource allocation, including user scheduling and power assignment, is conducted in a distributed manner to serve as many users in the whole network as possible. In the second step, based on the resource allocation scheme, the optimization objective namely network resource efficiency can be calculated and load balancing is implemented by switching the user that can maximize the objective function. Lagrange Multipliers method and heuristic algorithm are used to resolve the formulated optimization problem. Simulation results show that our algorithm achieves better performance in terms of user throughput, fairness, load balancing index and unsatisfied user number compared with the traditional approach which takes resource allocation and load balancing into account, respectively.
\end{abstract}

\section{Keywords}

Load balancing, resource allocation, LTE SON, efficiency and fairness

\section{Introduction}

Mobile operators are facing two challenges in the next generation of wireless communication technology: the ever-expanding demand for wireless access and the coexistence of heterogeneous network, wherein thousands of nodes and parameters should be taken into consideration. To address this problem, the technologies of Orthogonal Frequency Division Multiple Access (OFDMA) and SelfOrganizing Networks (SON) have been introduced by the 3rd Generation Partnership Project (3GPP) Long Term Evolution (LTE) to ensure superior mobile service while reducing manual intervention. The OFDMA is adopted as the downlink access technology in LTE system for its outstanding performance such as high spectrum efficiency and the ability to eliminate intra-cell interference, etc. The concept of self-organizing network which including selfconfiguration, self-optimization and self-healing, is introduced aiming at reducing operational expenditures (OPEX) and improving user experience by automated mechanisms as well [1]. However, the network performance is still deteriorated due to unbalanced load distributions among cells and inefficient utilization of system resource. The overload cell not only fails to guarantee the Quality of Service (QoS), but may also cause severe inter-cell interference (ICI) for edge users in neighbor cells. Load balancing, one of the key technologies in self-optimization, refers to the process whereby distributing the load among the unequal load cells by tuning the cell handover parameters adaptively. In addition, the proper resource allocation scheme may accommodate as many users in the heavy load cell as possible by improving the resource efficiency, while insuring fairness between cell-edge and cell-center users.

Recently, numerous researches concentrate on load balancing or resource allocation in the multi-cell OFDMAbased network. A mathematic perspective of self-optimizing wireless network is proposed in [2], which introduces a mathematical model of load balancing. The cell load is defined by the ratio of the required physical resource blocks (PRB) and the available total PRBs in one cell [3]. The cell is viewed to be overloaded when the ratio is larger than 1. A traditional adaptive handover approach to achieve load balancing with load fluctuations is presented in [4]. The highest PRB-utilization cell is chosen as the source cell and the target cell is selected by the certain measurement mechanism called A3 events. Further relevant references focused on the load balancing in the literature are included in [5-11]. Utility-based adaptive radio resource allocation is studied in [12] and specific utility functions are used in the simulations. According to [15], a novel resource allocation method which includes PRB and power assignment is proposed to achieve a significant balanced performance improvement between cell-edge and cell-center users. In [16], the problem of joint allocation of receiver, bit, and power over subcarriers is formulated into a nonlinear mixed integer programming (MIP) problem and figured out by decomposing the joint problem into 
a set of smaller-scale linear MIPs.

It is noteworthy that, as far as we know, the influence of resource allocation scheme is seldom considered in the research of load balance. However, load balancing alone, i.e. selecting the appropriate cell-edge user to switch to target neighbor cell, is not enough to improve the user experience in the whole network. This is because improper Radio Resource Mechanism (RRM) may lead to inefficient utilization of system resource, which increases the cell load and unsatisfied user number in hot spots. In fact, load balancing is regarded as a significant RRM which is crucial to guarantee high system performance for OFDMA-based LTE networks. In this paper, resource allocation and load balancing are jointly processed to improve the network performance and user experience. We formulate it as a problem of network resource efficiency maximization, which is subject to inter-cell interference and intra-cell resource constraints. Resource allocation and load balancing are implemented separately in different stages of optimization. However, the joint resource allocation consisting of user scheduling and power assignment can be formulated as a MIP problem, which has been proven to be NPhard [17]. We use a heuristic algorithm based on Lagrange Multipliers approach to deal with the sub-optimization case. Another concerned issue of resource allocation is how to balance network throughput and user fairness. To tackle this problem, the presented scheme which is based on modified max carrier to interference ratio (C/I) makes full advantage of multi-user diversity to serve more users while guaranteeing fairness between cell-edge and cell-center users. After a long time collection of measuring results, a practical algorithm which includes handover decision and call admission control is conducted on the load balancing stage, aiming at maximizing the objective function.

The remainder of this paper is organized as follows. In Sec. 2, we introduce the system model and the formulation problem. Section 3 presents the proposed resource allocation schemes, including throughput-driven user scheduling and power optimization subject to both evolved Node B (eNB) power and each PRB power, respectively. A practical algorithm of load balancing to further improve the system optimization objective is given in Sec. 4. The performance improvement is demonstrated and analyzed by simulations in Sec. 5. Conclusions are drawn in Sec. 6.

\section{System Model and Problem Formulation}

\subsection{Network Model}

An OFDMA-based 3GPP LTE downlink multi-cell network with users of Constant Bit Rate (CBR) service is considered here. Each cell is controlled by a center eNB equipped with an omnidirectional antenna. The set of cells and users in the system are denoted by $C=\{1, \ldots \ldots, \mathrm{C}\}$ and $K=\{1, \ldots \ldots, \mathrm{K}\}$, respectively. Due to the plane architecture of LTE network, every eNB has to make a decision of resource allocation in each transmission time interval (TTI). Considering the tradeoff between system performance and time overhead of the simulation, the duration of a TTI mentioned in this paper is much longer than a subframe ( $1 \mathrm{~ms})$. Adjacent twelve sub-carriers in the frequency domain and one slot duration in the time domain are grouped into a PRB, which is regarded as the smallest unit that can be allocated to a user. All cells in the network share the same frequency band which can be divided into PRBs indexed by $M=\{1, \ldots \ldots, \mathrm{M}\}$.

\subsection{Link Model}

A binary assignment indicator function is defined as $I_{i, m, k} \in\{0,1\}$, where $I_{i, m, k}=1$ means that the $m$-th PRB in the $i$-th cell is assigned to the $k$-th user, and $I_{i, m, k}=0$ otherwise. According to the LTE agreement, each PRB within a cell cannot be assigned to more than one user in the same TTI to avoid intra-cell interference. So we have

$$
\sum_{k=1}^{\mathrm{K}} I_{i, m, k} \leq 1 \quad \forall i \in C, \forall m \in M
$$

Assuming that channel state information (CSI) is available at eNBs and each user knows the instantaneous signal strength from all neighboring cells through pilot detection. Channel condition is supposed to remain constant within a TTI. Then the instantaneous signal-to-interference-and-noise ratio ( SINR) of user $k$ on PRB $m$ in cell $i$ can be written as

$$
\operatorname{SINR}_{i, m, k}=\frac{p_{i, m, k} \cdot g_{i, m, k}}{N_{0}+\sum_{j \in C, j \neq i} \rho_{j} \cdot p_{j, m, k} \cdot g_{j, m, k}}
$$

where $p_{i, m, k}$ and $g_{i, m, k}$ represent the power allocation to user $k$ on PRB $m$ in cell $i$ and channel gain between user $k$ and cell $i$ on PRB $m$, respectively. If $I_{i, m, k}=0$, the transmit power $p_{i, m, k}=0$. The cell load is defined as the fraction of used PRBs and $\rho_{j}$ is the load of cell $j$. ICI arises when multiple adjacent cells operate on the same PRB. The closed form of ICI received by user $k$ in cell $i$ on PRB $m$ is expressed as $\sum_{j \in C, j \neq i} \rho_{j} \cdot p_{j, m, k} \cdot g_{j, m, k}$, abbreviated as $\gamma_{k}^{i}(p)$ for simplicity. The interference received by user $k$ in cell $i$ is a strictly increasing function of the load of neighbor cell $j \neq i . N_{0}$ is the thermal noise power spectral density. Due to (2), the achievable data rate of user $k$ on PRB $m$ in cell $i$ is

$$
\omega_{i, m, k}=B \cdot \log \left(1+\operatorname{SINR}_{i, m, k}\right)
$$

where $B$ is the bandwidth of each PRB. For users with 
CBR service in the network, the resource allocation should try to meet their rate requirement. It is stated as

$$
r_{k}=\sum_{m} \omega_{i, m, k} I_{i, m, k} \geq R_{k} \quad \forall i \in C, \forall k \in K
$$

where $r_{k}$ and $R_{k}$ represent available data rate and request data rate of user $k$, severally. Constraint (4) states that user $k$ can be served by cell $i$ which can provide a larger data rate than QoS requirement. The unsatisfied user denoted by $k_{u}$ is defined as the user whose available rate cannot meet the request data rate.

\subsection{Problem Formulation}

Our optimization goal is to maximize network resource efficiency subject to inter-cell interference and intra-cell resource constraints. The reason is that load imbalance and improper RRM often cause inefficient utilization of system resource, which results in a great number of unsatisfied users in overload cells. Due to the fact that OFDMA can eliminate intra-cell interference by assigning users to orthogonal subcarriers, equal power allocation over all PRBs is always used for implementation simplicity as well as analytical tractability to resolve downlink resource allocation problem [17]. However, the equal power allocation may be excessive for the users with good channel condition while insufficient for the cell-edge users with poor channel condition. Thus, the unsatisfied users could only turn to request more bandwidth resource. This may eventually lead to the cell load increases or even overload. In the case of the smallest radio resource unit has been stipulated by LTE, the finer granularity of power allocation, the better performance of the system. Our scheme should optimize the cell ability to serve more users subject to limited resources and furthermore distribute the load among the unequal load cells. Therefore, the optimization problem is formulated as

$$
\begin{array}{cc}
\text { (P) } \max \sum_{i \in C} \sum_{k \in K} \sum_{m \in M} u_{i, m, k}=\sum_{i \in C} \sum_{k \in K} \sum_{m \in M} \frac{\omega_{i, m, k} \cdot I_{i, m, k}}{p_{i, m, k} \cdot I_{i, m, k}} \\
\text { s.t. } \sum_{m} B \cdot \log \left(1+\frac{p_{i, m, k} \cdot g_{i, m, k}}{N+\gamma_{k}^{i}(p)}\right) \cdot I_{i, m, k} \geq R_{k} & \forall k \in K \quad \forall i \in C \\
\sum_{k} \sum_{m} p_{i, m, k} I_{i, m, k} \leq P_{B S} & \forall i \in C, \\
\sum_{k} p_{i, m, k} I_{i, m, k} \leq P_{s u b} & \forall i \in C \forall m \in M, \\
\sum_{m} \sum_{k} I_{i, m, k} \leq \mathrm{M} & \forall i \in C .
\end{array}
$$

$u_{i, m, k}$ indicates the earned profit, i.e. resource efficiency, if PRB $m$ in cell $i$ is allocated to user $k$.Constraint in (5) which is derived by (2), (3) and (4) represents that the available data rate of any user in its current serving cell must achieve its data requirement. $P_{B S}$ and $P_{s u b}$ in constraints (6) and (7) are the total power limitation at the eNB and each PRB, respectively. $M$ in constraint (8) denotes the total number of available PRBs in each cell. The solution of the problem is to achieve a better performance balance between resources and users, where all users can equally get desirable available data rates despite of their channel conditions and QoS requirements. This problem is known as NP-hard since the computational complexity of seeking out the optimal solution is in exponential order of the problem size. Hence we need to seek for a sub-optimal method.

\section{Resource Allocation Scheme}

The feasible scheme proposed in this section is implemented in two steps. In the first step, an efficient resource allocation, including user scheduling and power assignment, is conducted in a distributed manner to serve as many users in the whole network as possible. In the second step, based on the resource allocation scheme mentioned in the first step, the network resource efficiency can be calculated and load balancing is implemented by switching the user that can maximize the objective function. As the plane architecture of LTE network without Radio Network controller (RNC), the resource allocation has to be decided individually within each cell in a distributed manner. Neighbor eNBs exchange their ICI and load status information periodically through X2 interface. A concerned issue of resource allocation is how to balance network throughput and user fairness. In order to take full advantage of multi-user diversity to insure resource efficiency while balancing fairness among users with different channel conditions, a heuristic algorithm based on the Lagrange Multipliers approach is used to solve the problem $(P)$ described above. Then the resource optimization problem can be changed into an unconstrained optimization problem expressed as:

$$
\max \left(\sum_{k \in K} \sum_{m \in M} I_{i, m, k}\left(u_{i, m, k}-\lambda_{m} p_{i, m, k}\right)\right) \quad \forall i \in C
$$

where $\lambda_{m}, m=1,2 \cdots \cdots \mathrm{M}$ are $\mathrm{M}$ non-negative Lagrange Multipliers which are initialized to be zero, and $u_{i, m, k}$ is the resource efficiency if PRB $m$ in cell $i$ is allocated to user $k$. The feasible solution of sub-optimization problem can be achieved if all $\lambda_{m}$ are calculated and updated to satisfy the constraint equation (6). Firstly, a modified max $\mathrm{C} / \mathrm{I}$ scheme is adopted to allocate PRB $m$ in cell $i$ to the only unsatisfied user $k=\arg \max _{k} \operatorname{SINR}_{i, m, k}$. Then the request power of user $k$ to guarantee the QoS can be calculated by $p_{i, m, k}=\frac{\left(2^{\frac{R_{k}-\mathrm{r}_{k}}{B}}-1\right) \cdot\left(N+\gamma_{k}^{i}(p)\right)}{g_{i, m, k}}$ under the condition of (7). If the sum of the allocated power is larger than the eNB power constraint, in order to update the 
Lagrange multipliers efficiently, the PRB with the biggest power will be found and reallocated, which leads to the minimum Lagrange multiplier increase until the sum of the allocated power satisfy the constrain (6). If there are multiple PRBs with same maximum power in a TTI, we will choose the one which correspond to the user with the lowest available date rate to be reallocated. The proposed resource allocation scheme can improve system performance effectively. On one hand, the PRB assignment based on modified max $\mathrm{C} / \mathrm{I}$ can guarantee radio resource efficiency and network throughput, on the other hand, the flexible power allocation scheme can improve the fairness among users by avoiding excessive power assignment to cell center users compared with traditional equal power allocation proposal.

Algorithm 1 Pseudocode of the algorithm

1: for $i=1: \mathrm{C}$

2: Initial the Lagrange multipliers, $\lambda_{m} \longleftarrow 0$, for all $m$

3: Pre-assignment phase

4: $\quad k \longleftarrow \arg \max _{k_{u}}\left\{\operatorname{SINR}_{i, m, k}\right\}, I_{i, m, k} \longleftarrow 1$, for all $m$

5: $\quad p_{i, m, k}= \begin{cases}\frac{\left(2^{\frac{R_{k}-\mathrm{r}_{k}}{B}}-1\right) \cdot\left(N+\gamma_{k}^{i}(p)\right)}{g_{i, m, k}} & \text { if } p_{i, m, k} \leq P_{s u b} \\ P_{s u b} & \text { else }\end{cases}$

6: Calculate the total allocated power violation

7: $\quad \varepsilon_{i} \longleftarrow P_{B S}-\sum_{m=1}^{\mathrm{M}} \sum_{k=1}^{\mathrm{K}} p_{i, m, k} I_{i, m, k}$

8: $\quad$ while $\varepsilon_{i}<0$

9: $\quad$ for $m=1: M$

10: $\quad$ for $k=1: K$

11: $\quad P_{\max } \longleftarrow \max \left\{p_{i, m, k}\right\}$

12: $\quad m^{*}, k^{*} \longleftarrow \arg \min _{m^{\prime}, k}\left\{r_{k^{\prime}}\right\} \mid m^{\prime} \longleftarrow \underset{m}{\arg }\left\{p_{i, m, k}=P_{\max }\right\}$

$k_{m^{\prime}}$ is the user corresponding to $\operatorname{PRB} m^{\prime}$

13: $\quad$ end for

14: $\quad$ end for

\section{5: Reallocation}

16: for $k=1: K$

17: $\Delta_{k} \longleftarrow \mu_{i, m^{*}, k^{*}}-\mu_{i, m^{*}, k}-\lambda_{m^{*}}\left(p_{i, m^{*}, k^{*}}-p_{i, m^{*}, k}\right)$

18: $\quad$ end for

19: $\hat{k} \longleftarrow \arg \min _{k}\left\{\Delta_{k}\right\}$, for all $k$

20: Update the Lagrange multiplier, I and $\varepsilon_{i}$
21:

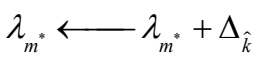

22:

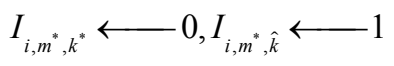

23:

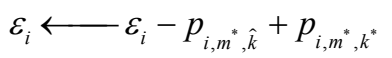

24: end while

25: end for

\section{Load Balancing Algorithm}

After a long time user measuring and data collecting, a practical algorithm including handover decision and call admission control is conducted on the load balancing stage. Just as resource allocation, load balancing is implemented in a distributed way using limited neighbor information through X2 interface. Since we are trying to maximize the objective function, the value of the network resource efficiency after handover should be larger than that before. Assuming that user $k$ is switched from cell $i$ to cell $j$, the following constraint should be satisfied

$$
\sum_{m \in M_{k}} \frac{\omega_{j, m, k}}{p_{j, m, k}}-\sum_{m_{k} \in M} \frac{\omega_{i, m, k}}{p_{i, m, k}}>0
$$

where $M_{k}$ denotes the set of PRBs which is allocated to user $k$ within a certain TTI. The left part of inequality can be defined as handover gain $G_{i, j}^{k}$. Because the period of load balance is much longer than the resource allocation cycle, our proposed scheme adopts average handover gain which needs to collect data for a relative long time to eliminate the influence of the instantaneous channel fading. The left part of inequality (10) is rewritten as the following form

$$
\overline{G_{i, j}^{k}}=\frac{1}{T} \sum_{t=1}^{T}\left(\sum_{m \in M_{k}} \frac{\omega_{j, m, k}(t)}{p_{j, m, k}(t)}-\sum_{m \in M_{k}} \frac{\omega_{i, m, k}(t)}{p_{i, m, k}(t)}\right) .
$$

In constraint (11) $T$ is the statistics period. To guarantee the stability of the heuristic algorithm, we only switch the user with the maximal average handover gain in the whole network during each load balancing cycle. Moreover, the candidate handover user should also satisfy the call admission control of the target cell, or we have to select the subprime user until the one who can meet all handover conditions.

$$
\begin{gathered}
\overline{G_{i, j}^{k}}>0, \\
\rho_{j}+\rho_{j, k} \leq 1, \\
\operatorname{SINR}_{j, k} \geq \operatorname{SINR}_{T h} .
\end{gathered}
$$

The load of user $k$ in target cell $j$ is denoted by $\rho_{j, k}$. Equation (12) presents the any implemented handover 
should increase the network resource efficiency. Constraint in (13) expresses that the target cell should have enough spare resource for all existing users and the switching one. Constraint in (14) tells that the SINR detected in target cell by user $k$ should be larger than its access threshold $\operatorname{SINR}_{T h}$.

\section{Performance Evaluation}

Simulations are used here to evaluate the performance of the proposed joint dynamic radio resource allocation and mobility load balancing scheme in LTE downlink networks.

\subsection{System Configuration}

A regular hexagonal of seven-cell network topology is considered with wrap-around technology to avoid border effect. Each cell is controlled by a center eNB with no sectors divided. Assuming the minimum data rate to ensure the QoS for every user is $720 \mathrm{Kbps}$. In terms of typical CBR service class such as voice and video, as long as the average speed (assuming that average during a relative short time interval) remains constant, the short-term fluctuation of data rate is usually not seemed to be a problem. Thus, an appropriate increase in the request rate which is set to be $1000 \mathrm{kbps}$ in our simulation can compensate for the instantaneous fading of time-varying channel. We set the TTI duration and load balancing cycle to be $0.1 \mathrm{~s}$ and $15 \mathrm{~s}$, respectively. Long time, for example, hour level simulation is needed to evaluate the proposed scheme.

\begin{tabular}{|c|c|}
\hline Parameter & Value \\
\hline Cell layout & 7 cells with wrap around technique \\
\hline User No. of center cell & 45 (40 users won't move out) \\
\hline User No. of other cells & 5 \\
\hline UE speed & $5 \mathrm{~km} / \mathrm{h}$ \\
\hline Cell radius & $1 \mathrm{~km}$ \\
\hline eNB Tx power & $46 \mathrm{dBm}$ \\
\hline $\begin{array}{c}\text { Pathloss } \\
\text { Shadowing }\end{array}$ & $\begin{array}{c}114.8367+33.7717 * \log _{10}(d), d \text { in km } \\
\text { Log-normal with standard deviation } 8 \mathrm{~dB}\end{array}$ \\
\hline $\begin{array}{c}\text { PRB bandwidth } \\
\text { Traffic model }\end{array}$ & $180 \mathrm{kHz}$ \\
\hline CoS guarantee data rate & $720 \mathrm{Kbps}$ \\
\hline Request data rate & $1000 \mathrm{Kbps}$ \\
\hline Resource allocation period & $0.1 \mathrm{~s}$ \\
\hline $\begin{array}{c}\text { User satisfaction } \\
\text { measurement cycle }\end{array}$ & $5 \mathrm{~s}$ \\
\hline Load balancing cycle & $15 \mathrm{~s}$ \\
\hline
\end{tabular}

Tab. 1. Simulation parameters.

In order to simulate the load balancing scenario and differentiate the loads of neighboring cells, we set the center cell of the network to be the overloaded one with 45 users distributed uniformly within it. Each user engages a random walk with correlation distance of $20 \mathrm{~m}$ and changes direction with the maximum update angle of 45 degrees. Supposing that in order to maintain the heavy load concentration, 40 users in center cell would not move out of the original cell. The channel model reference COST 231-Hata in which the frequency of transmission is modified to $3500 \mathrm{MHz}$ to apply to this scenario. The main simulation parameters are given in Tab. 1 .

\subsection{Simulation Results}

Firstly, we evaluate the performance of the proposed resource allocation algorithm in terms of cumulative distribution for user throughput in each TTI and user fairness index. There are two reference scenarios, equal power allocation among PRBs and Fractional Frequency Reuse (FFR) scheme. The proposed load balancing algorithm is adopted by all of them.

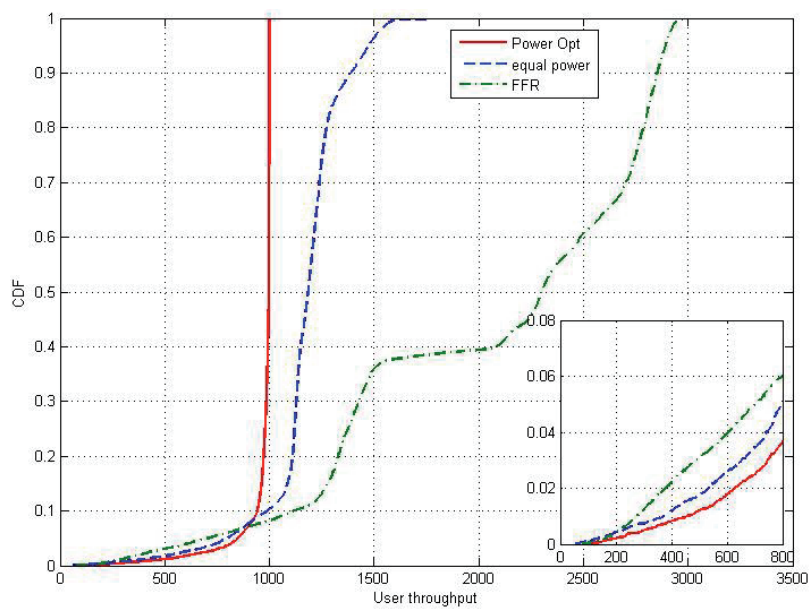

Fig. 1. Cumulative distribution for user throughput.

Figure 1 shows the cumulative distribution function (CDF) of user throughputs achieved in each TTI by different schemes for the same user distribution in the reference network. On one hand, we can find that the vast majority of user throughputs are very close to $1000 \mathrm{Kbps}$ in our proposed scheme. On the other hand, in the traditional equal power allocation and FFR scheme, user throughputs span a large range. It is because that too much frequency and power resources are occupied by the good channel condition users, who achieve larger available data rate than their actual QoS needs. Therefore, the available resources left for cell-edge users with poor channel condition become relative less. If the wireless resources are abundant enough, user throughput is the bigger the better and the FFR will be the best choice of the three schemes. However, in load balancing scenario, making effort to guarantee QoS for all users in the network is the most significant. Fig.1 reveals that more than 3\% users cannot achieve QoS guaranteed data rate of $720 \mathrm{Kbps}$ in both reference scenarios while in the proposed algorithm there are about $2 \%$ unsatisfied users. If the system users in each TTI can be regarded as independent irrelevant, the proposed scheme can obviously reduce the number of unsatisfied users in the network during the whole simulation. 


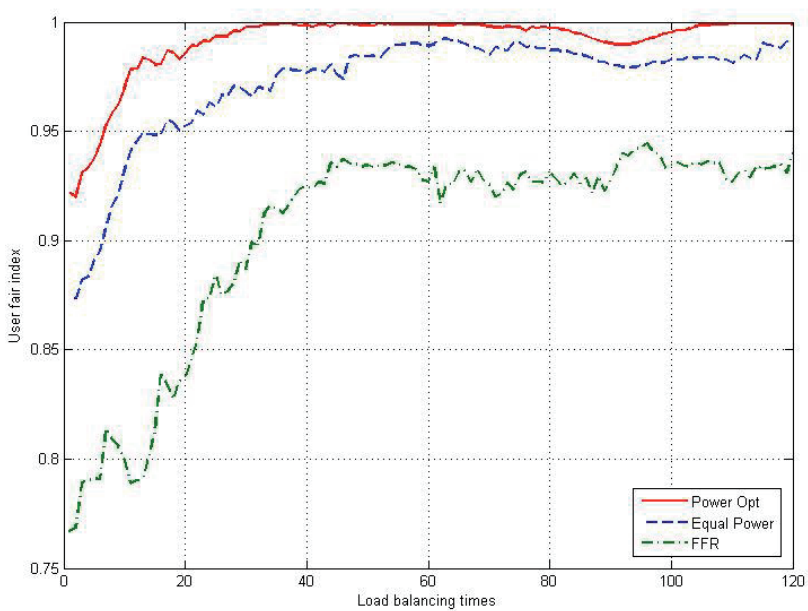

Fig. 2. User fairness index.

Figure 2 shows the user fairness index versus load balancing times. The index is defined as $F=\frac{\left(\sum_{k \in K} r_{k}\right)^{2}}{\mathrm{~K}\left(\sum_{k \in K} r_{k}^{2}\right)}$, which ranges in $[1 / \mathrm{K}, 1]$. A larger

$F$ denotes a fairer throughput distribution among users. We can observe that the user fairness in the proposed resource allocation algorithm outperforms the other two schemes significantly throughout the simulation time. This is because the proposal can avoid excessive resource allocation to cell-center users and leave more resource to help users with poor channel condition to achieve a lager throughput so as to improve the fairness among all users in the system.

We can find that the proposed resource allocation scheme can achieve better performance in terms of guaranteeing the user QoS and the user fairness index from the above results. Thus, our proposed resource allocation scheme is selected for the following simulations.

Based on the resource allocation scheme discussed above, the optimization objective representing network resource efficiency can be calculated. Then we focus on the practical load balancing algorithm in terms of cumulative distribution for user throughput in each TTI, load balancing index and the number of unsatisfied users. The no load balancing scenario and a conventional MLB method presented in [4] are used for reference.

Figure 3 shows the cumulative distribution function (CDF) of user throughput achieved in each TTI by the different schemes. The throughputs in all scenarios converge to $1000 \mathrm{Kbs}$. However, the proposed LB algorithm can successfully maintain high performance for almost all users regardless of channel equality, i.e., if the system users in each TTI can be regarded as independent irrelevant, more than 95\% users can achieve throughput over $720 \mathrm{Kbps}$ compared to about $90 \%$ users in the MLB case.

Figure 4 shows the load balancing index varying with load balancing times. Load balancing index is defined as $\varepsilon(t)=\frac{\left[\sum_{i \in C} \rho_{i}(t)\right]^{2}}{\mathrm{C}\left[\sum_{i \in C} \rho_{i}^{2}(t)\right]}$. Just as the user fairness index, a larger

$\varepsilon(t)$ denotes a more balanced load distribution among cells. The load balancing index in proposed LB case increases from the initial value 0.67 to the value close to 1 with the

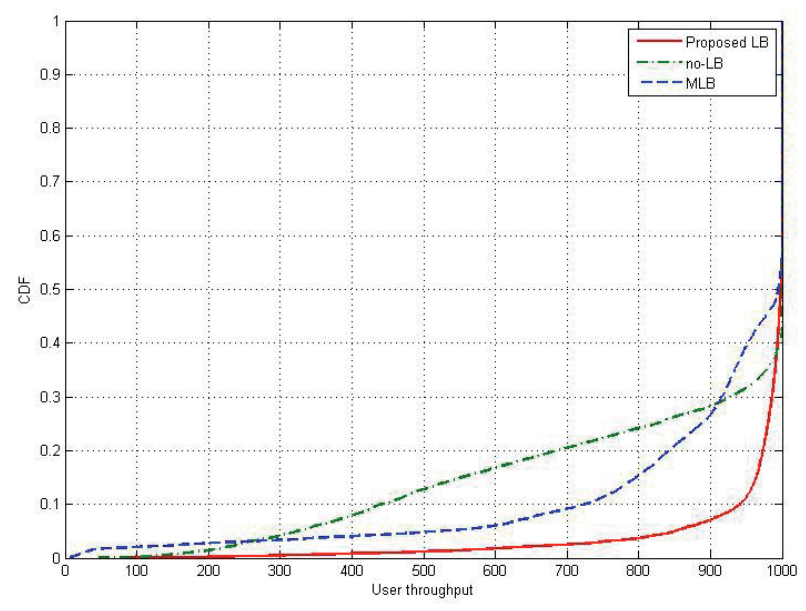

Fig. 3. Cumulative distribution for user throughput in LB scenario.

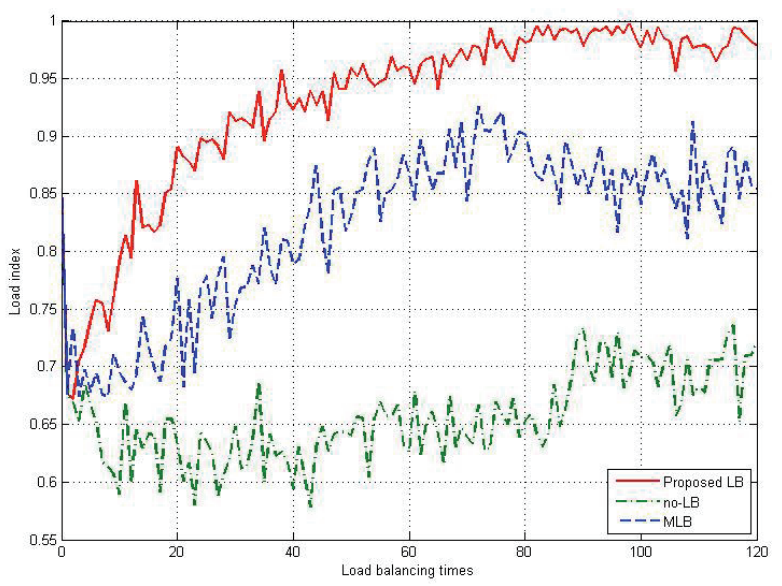

Fig. 4. Network load index.

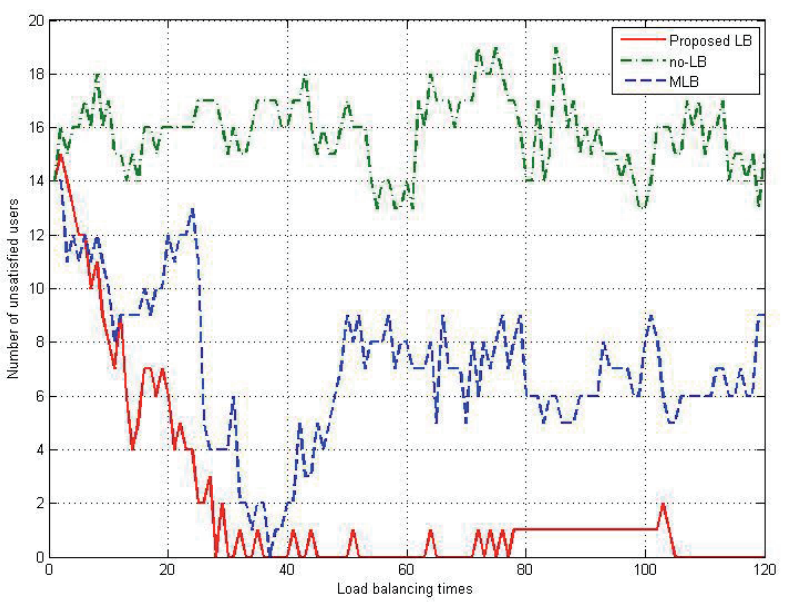

Fig. 5. Total number of unsatisfied users in overall network. 
increase of load balancing times, representing the traffic distribution in the whole network becomes more and more uniform. The result for MLB method is inferior and the case of no-LB is as poor as expected.

Figure 5 shows the number of unsatisfied users versus load balancing times. We can observe a significant number of unsatisfied users which is always hovering around 16 out of 75 in the no load balancing scenario. Using MLB can improve user satisfaction. However, MLB method is difficult to keep a stable performance. In the LB case, the number of unsatisfied users decreases dramatically to about 1 after 25 load balancing times, and the good performance can be maintained to the end of simulation.

\section{Conclusion}

In this paper, joint dynamic radio resource allocation and mobility load balancing are considered in 3GPP LTE downlink OFDMA-based network. The optimization problem is formulated as maximizing network resource efficiency subject to inter-cell interference and intra-cell resource constraints. Resource allocation and load balancing are implemented in different stages of optimization. A heuristic algorithm based on Lagrange Multipliers approach is used in user scheduling and power optimization. The scheme makes full advantage of multiuser diversity to guarantee resource efficiency and network throughput while improving fairness between cell-edge and cell-center users. Then a practical load balancing algorithm is proposed to further improve the optimization objective. Simulation results demonstrate that the proposed scheme can significantly optimize the network performance in terms of user throughput, user fairness, load balancing index and the number of unsatisfied users.

\section{Acknowledgements}

This work was supported in part by the National Natural Science Foundation of China under Grant 11131006,61075054 and 11301413 , by the International Cooperation and Exchanges Project under Grant S2010GR0902, and China Postdoctoral Science Foundation under Grant 2012M521774.

\section{References}

[1] TR 36.902, 3RD GENERATION PARTNERSHIP PROJECT. Technical Specification Group Radio Access Network; Evolved Universal Terrestrial Radio Access (E-UTRA); Self-configuring and self-optimizing network use cases and solutions; Protocol specification (Release 9).

[2] VIERING, I., DOTTLING, M., LOBINGER, A. A mathematical perspective of self-optimizing wireless networks. In IEEE International Conference on Communications ICC'09. Dresden (Germany), 2009, p. 1-6.
[3] LOBINGER, A. STEFANSKI, S., JANSEN, T., BALAN, I. Load balancing in downlink LTE self-optimizing networks. In IEEE $71^{\text {th }}$ Vehicular Technology Conference. Taipei (Taiwan), 2010, p. 1-5. DOI: $10.1109 /$ VETECS.2010.5493656

[4] KWAN, R., ARNOTT, R., PATERSON, R., TRIVISONNO, R., KUBOTA, M. On mobility load balancing for LTE systems. In IEEE $72^{\text {th }}$ Vehicular Technology Conference. Ottawa (Canada), 2010, p. 1-5. DOI: 10.1109/VETECF.2010.5594565

[5] ZHANG, L., LIU, Y., ZHANG, M. G., JIA, S. C., DUA, X. Y. A two-layer mobility load balancing in LTE self-organization networks. In Proceedings of IEEE $13^{\text {th }}$ International Conference on Microwave Conference on Communication Technology. Jinan (China), 2011, p. 925-929. DOI: 10.1109/ICCT.2011.6158014

[6] HU, H. L., ZHANG, J., ZHENG, X. Y., YANG, Y., WU, P. Selfconfiguration and self-optimization for LTE networks. IEEE Communications Magazine, 2010, vol. 48, no. 2, p. 94-100. DOI: 10.1109/MCOM.2010.5402670

[7] LI, Z. H., WANG, H., PAN, Z. W., LIU, N., YOU X. H. Joint optimization on load balancing and network load in 3gpp lte multi-cell networks. In Proceedings of International Conference on Wireless Communications and Signal Processing WCSP'2011. Nanjing (China), 2011, p. 1-5. DOI: 10.1109/WCSP.2011.6096768

[8] TONGUZ, O. K., YANMAZ, E. The mathematical theory of dynamic load balancing in cellular networks. IEEE Transactions on Mobile Computing, 2008, vol. 7, no. 12, p. 1504-1518. DOI: 10.1109/TMC.2008.66

[9] GIOVANIDIS, A., LIAO, Q., STANCZAKY, S. A distributed interference-aware load balancing algorithm for LTE multi-cell networks. In International ITG Workshop on Smart Antennas. Dresden (Germany), 2012, p. 28-35. DOI: 10.1109/WSA.2012.6181222

[10] SANG, A., WANG, X., MADIHIAN, M., GITlin, R. D. Coordinated load balancing, handover/cell-site selection, and scheduling in multi-cell packet data systems. Wireless Networks, 2008, vol. 14, no. 1, p. 103-120. DOI: 10.1145/1023720.1023750

[11] YANG, Y., DONG, W. X., CHEN, X. H., et al. A utility-based load-balancing scheme with guarantee of users' rate for LTE selforganization networks. International Journal of Network Management, 2013, vol. 23, p. 172-185. DOI: 10.1002/nem.1824

[12] KATOOZIAN, M., NAVAIE, K., YANIKOMEROGLU, H. Utility-based adaptive radio resource allocation in OFDM wireless networks with traffic prioritization. IEEE Transactions on Wireless Communications, 2009, vol. 8, no. 1, p. 66-71. DOI: 10.1109/TWC.2009.080033

[13] ZHANG, H. H., RANGARAJAN, S. Joint load balancing, scheduling, and interference mitigation in multi-cell and multicarrier wireless data systems. In $7^{\text {th }}$ International Symposium on Modeling and Optimization in Mobile, Ad Hoc, and Wireless Networks. Seoul (South Korea), 2009, p. 1-10. DOI: 10.1109/WIOPT.2009.5291637

[14] SON, K., CHONG, S., VECIANA, G. Dynamic association for load balancing and interference avoidance in multi-cell networks. IEEE Transactions on Wireless Communications, 2009, vol. 8, no. 7, p. 3566-3576. DOI: 10.1109/TWC.2009.071140

[15] YU, Y. W., DUTKIEWICZ, E., HUANG, X. J., MUECK, M. Downlink resource allocation for next generation wireless networks with inter-cell interference. IEEE Transactions on Wireless Communications, 2013, vol. 12, no. 4, p. 1783-1793. DOI: $10.1109 /$ TWC.2013.030413.120760

[16] FATHI, M., KARIPIDIS, E. Distributed resource optimization in multicell OFDMA networks. In Proceedings of IEEE Wireless Communications and Networking Conference. Shanghai (China), 2012, p. 1316-1320. DOI: 10.1109/WCNC.2012.6213982

[17] LI, G. Q., LIU, H. Downlink radio resource allocation for multi- 
cell ofdma system. IEEE Transactions on Wireless Communications, 2006, vol. 5, no. 12, p. 3451-3459. DOI: 10.1109/TWC.2006.256968

[18] SHEN, Z. K., ANDREWS, J. G., EVANS, B. L. Adaptive resource allocation in multiuser OFDM systems with proportional rate constraints. IEEE Transactions on Wireless Communications, 2005, vol. 4, no. 6, p. 2726-2737. DOI: 10.1109/TWC.2005.858010

[19] JANG, J. H., LEE, K. B. Transmit power adaptation for multiuser OFDM systems. IEEE Journal on Selected Areas in Communications, 2003, vol. 21, no. 2, p. 171-178. DOI: 10.1109/JSAC.2002.807348

[20] VISWANATH, P., TSE, D. N. C., ANANTHARAM, V. Asymptotically optimal water-filling in vector multiple-access channels. IEEE Transactions on Information Theory, 2001, vol. 47, no. 1, p. 241-267. DOI: $10.1109 / 18.904525$

\section{About the Authors ...}

Feng LI was born in 1981. He received his B.Sc. and Ph.D. degrees in 2003 and 2009, respectively, both from Xi'an Jiaotong University. He is now a lecturer in the same university. His research interests include statistical signal processing and wireless communications.

Lina GENG was born in Xi'an, China, in 1989. She received her B.Sc. from Northwest University, Xi'an, China, in 2011. Now she is working towards her M.Sc. in School of Electronics and Information Engineering of Xi'an Jiaotong University. Her research interests include SelfOrganization Network and resource allocation.

Shihua ZHU was born in 1950. He received the B.Sc. degree of Radio Engineering in 1982 from Xi'an Jiaotong University, China. After that, he received the M.Sc. degree of Telecommunication Systems in 1984, and Ph.D. degree of Electronic Systems Engineering in 1987 in the University of Essex, UK. Since 1987, he has been with Xi'an Jiaotong University where he is now a professor in the Department of Information and Communications Engineering. His research interests are broad in the area of mobile communications, digital transmission and broadband networks. 